\title{
TRANSPERITONEAL URETERCUTANEOSTOMY WITH USE OF MESOSIGMOID COLON PERITONEUM IN PATIENTS WITH BLADDER CANCER AFTER RADICAL CYSTECTOMY
}

DOI: 10.36740/WLek202005128

\author{
Oleksandr E. Stakhovskyi, Maksym V. Pikul, Sofia L. Semko, luriy V. Vitruk, Oleg A. Voylenko, \\ Oleksiy A. Kononenko, Bogdan V. Grechko, Eduard 0. Stakhovsky \\ DEPARTMENT OF PLASTIC AND RECONSTRUCTIVE ONCOUROLOGY, NATIONAL CANCER INSTITUTE, KYIV, UKRAINE
}

\begin{abstract}
The aim of the study was to we describe the new surgical approach of constructing single site transperitoneal cutaneostomy with the use of sigmoid colon accompanied by skin modified fixation technique and its clinical outcomes.

Materials and methods: 89 patients were included in a single center study from January 2015 to May 2019. All patients underwent single-site modified transperitonial ureterocutanesotomy. Clinical and surgical data were analyzed. Statistical analyses were performed using SPSS 22.0.

Results: Majority of the patients had a low performance status and rationale for cystectomy was palliative cytoreduction. Almost half of the patients had upper tract obstruction at diagnosis with $40 \%$ of patients presenting with a decreased kidney function. Surgical procedure was safe taking to account locally advanced. All the surgeries were performed with acceptable complications rate. Blood loss didn't exceeded $1000 \mathrm{ml}$ and median operation time was 194 minutes. Early and late postoperative complications were analyzed and stomal stenosis that needed continuous restenting or reoperation was seen in $20 \%$ of cases.

Conclusions: Single-site modified transperitonial ureterocutanesotomy is safe and effective surgical approach with acceptable complication rates and duration. Such surgery may be used as a major urinary diversion approach in advanced bladder cancer patients with severe symptoms and low-performance status. Further external validation studies needed to evaluate efficacy of the proposed technique.
\end{abstract}

KEY WORDS: Bladder cancer, Radical cystectomy, Ureterocutanesotomy, Urinary diversion, Surgical technique

Wiad Lek. 2020;73(5):988-993

\section{INTRODUCTION}

Radical cystectomy (RC) remains the gold standard in treatment of muscle invasive bladder cancer. The question of urine diversion is already resolved with ileal reconstruction methods throughout decades, although they are not feasible for every case [1]. The major problem is derived from the fact that co-morbid or elderly patients have a higher risk of bowel-associated early complications that may affect 30-day mortality rate [2-3]. The simplest way of urine diversion is cutaneous ureterostomy that could potentially minimize complication rate and preserve life quality, although it is characterized by high incidence of ureteral stenosis [4-6].

Various attempts to minimize risks of ureteral stenosis discovered factors that potentially may affect ureteral obstructive changes: length of abdominal channel, preservation of blood supply, fibrous compression by intraperitoneal structures, ureteral kinking and tension [7-9]. Knowledge was successfully implemented into daily practice in different centers with different surgical variations. Attention was mainly devoted to minimizing abdominal channel length, preserving parietal peritoneum, shortening retroperitoneal or peritoneal way and wrapping ureter with omentum to improve its blood circulation. Nevertheless, there is no standardly approved procedure and results from different centers may vary.

In elderly and co-morbid patients kidney function is crucial, thus diversion type should be safe with account of acute kidney injury, upper urinary tract obstruction and occurrence of pyelonephritis. Catheter-free rate is probably one of the main parameters that count in this diversion group, because casual re-stenting induces inflammation, increases treatment-related costs and induces deterioration of kidney function. Bricker diversion (ileal conduit) also looks not ideal in this setting, because except risk of early bowel complications it can also lead to stoma or ureteral obstruction, which negatively affects filtration rate.

In this study we describe the new surgical approach of constructing single site transperitoneal cutaneostomy with the use of sigmoid colon accompanied by skin modified fixation technique and its clinical outcomes.

\section{THE AIM}

The aim of the study was to we describe the new surgical approach of constructing single site transperitoneal cutaneostomy with the use of sigmoid colon accompanied by skin modified fixation technique and its clinical outcomes. 


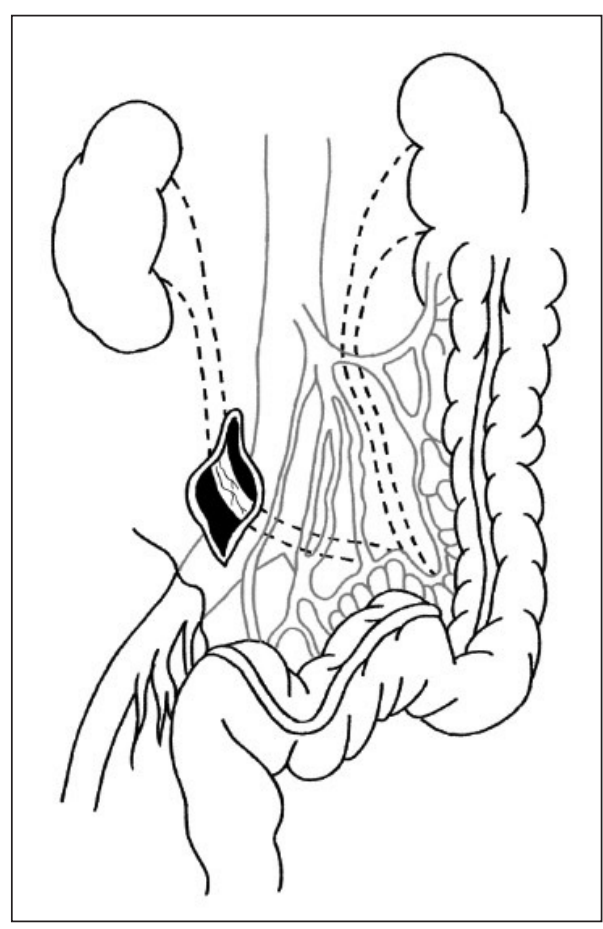

Fig. 1. Schematic view of the ureters path in the colon mesentery.

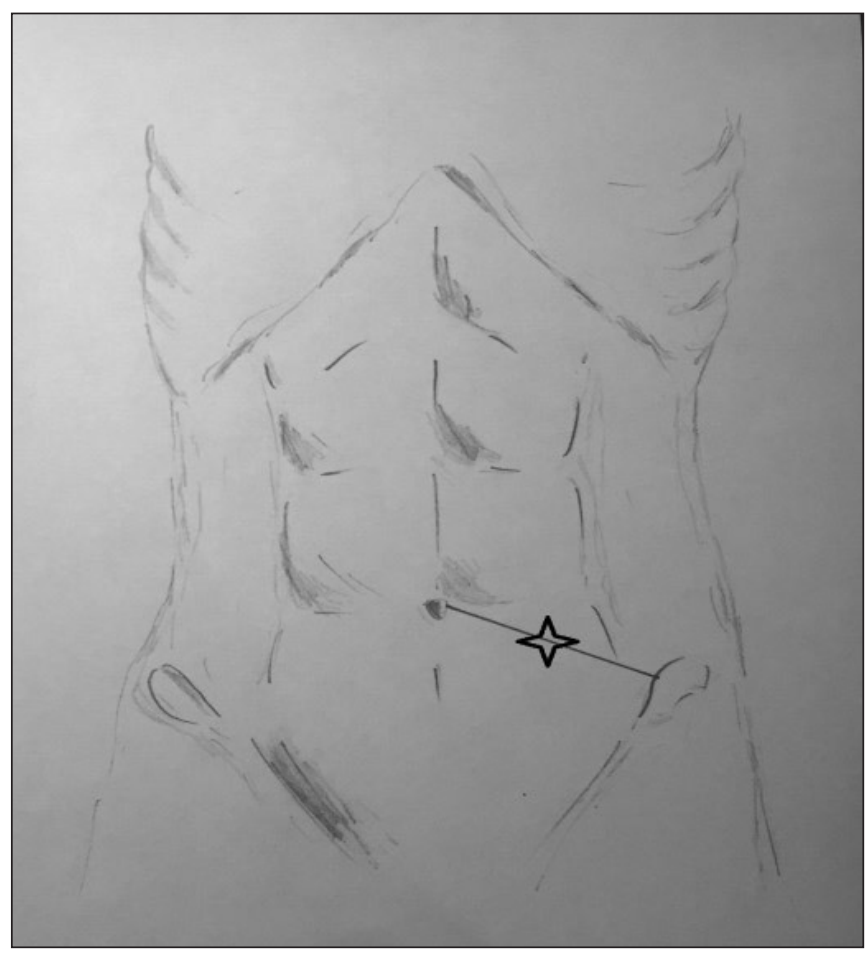

Fig. 3. Place of the ureters opening on the left abdominal wall.

\section{MATERIALS AND METHODS}

Overall, 89 patients were included in the study from January 2015 to May 2019. All patients underwent single-site modified transperitonial ureterocutanesotomy. The advantages and disadvantages of existing urinary diversion technique were explained to all patients and written consents were obtained from them. Surgical technique was initially performed in 12 patients after ethic committee agreement in

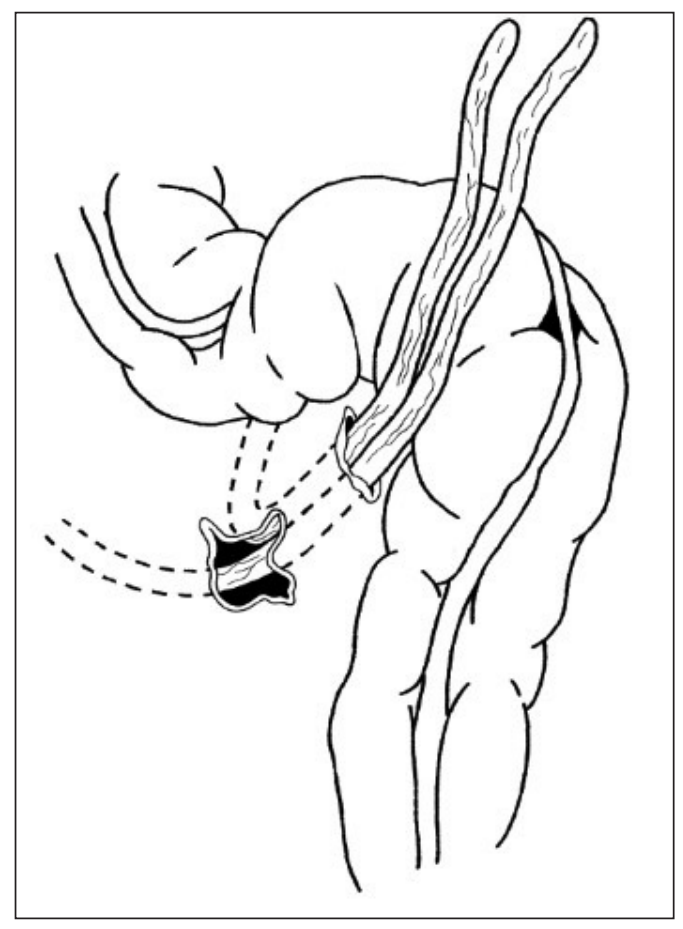

Fig. 2. Opening of the ureters tunnel in the mesosigmoid at the distance of $2 \mathrm{~cm}$ from colon.

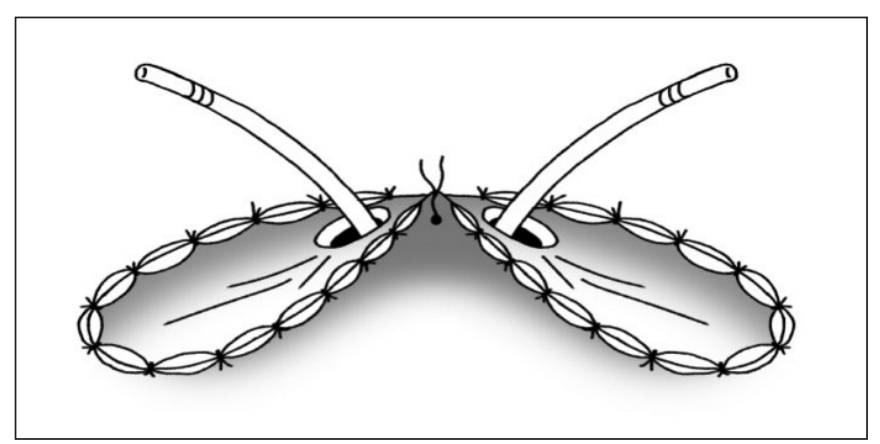

Fig. 4. Schematic view of the ureters anastomosed with the skin in modified X-shaped technique forming single-site cutaneous ureterostomy and preventing stomal stenosis.

terms of pilot clinical trial. After receiving satisfactory primary results method was agreed by local patent committee for further implementation and study design was approved by local ethic committee of National Cancer Institute.

The study included patients with advanced bladder cancers with high ASA-scoring (grade III and higher) or those undergoing surgery due to imperative indications. Five surgeons in two hospitals performed all the surgical procedures. Pre-surgical preparation and post-surgical follow-up was mainly based on ERAS protocols. Complications developed during hospital stay and the first 30 days of postoperatively were considered as early complications, while those occurring later than 30 days were considered as late complications. Catheter-free rate was calculated in all patients who had a minimum follow-up period for more than 12 months. From 89 patients only 5 were lost of follow-up during observation period. Follow-up visits were conducted every 3 months during the first year and every sixth month thereafter. 


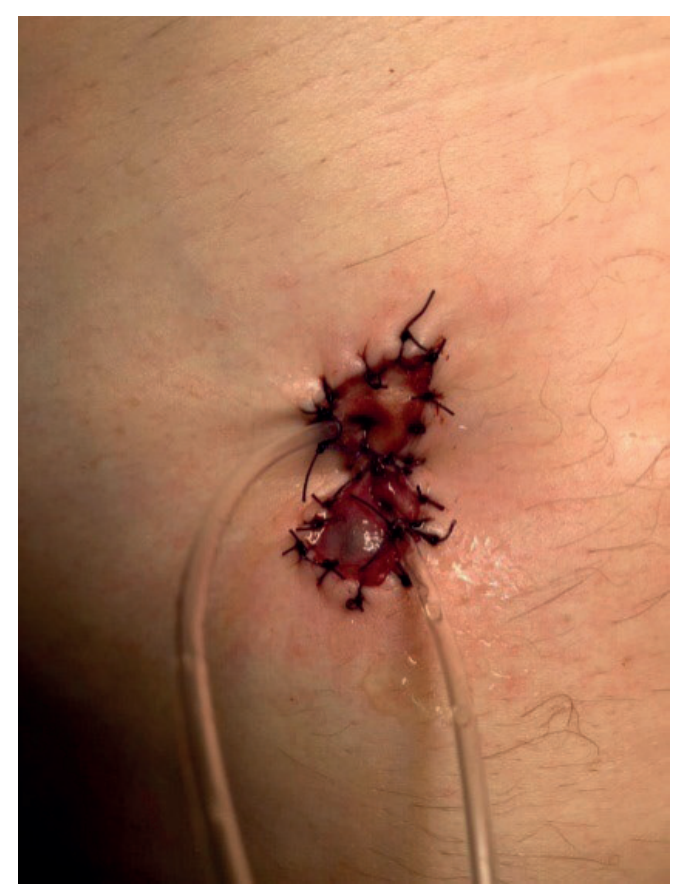

Fig. 5. Ureters anastomosis with skin, with two 6 Fr.stents inserted.

All patients were trained to change stomal pouches and were checked monthly by local urologists according to specially developed patients' cards for better complication rate monitoring. Every follow-up visit included standard computed tomography (to evaluate oncological and functional outcome), eGFR evaluation and stoma checking for obstruction.

\section{SURGICAL TECHNIQUE}

All 89 patients underwent standard volume of radical cystectomy. Extended lymph node dissection was performed only in cases of enlarged lymph nodes beyond template lines. Both ureters were used in all cases. Mobilization of the ureters was performed in manner to preserve sufficient peri-ureteric connective tissue. Ureteral preservation was crucial to spare as much length as possible. Negative margin was proved by frozen section during surgery.

After cystectomy, both ureters were freed cranially closer to the kidneys. In cases of kinking, arms were deliberated for optimal straightening. Sigmoid colon was mobilized medially by dissecting fixation of parietal peritoneum on the left. A window was created in the root of sigmoid colon mesentery, both ureters were brought retroperitoneally: the left one penetrating the mesentery between colon vessels heading the window and the right one under visceral peritoneum (figure 1). Then, the tunnel was formed beneath the visceral peritoneum of the mesosigmoid, which distally opened at the distance of $2 \mathrm{~cm}$ from colon (figure 2). Both ureters were passed throughout this tunnel and their ends were exteriorized through the opening in the left abdominal wall - anti-McBurney point (figure 3). Abdominal wall channel was cut

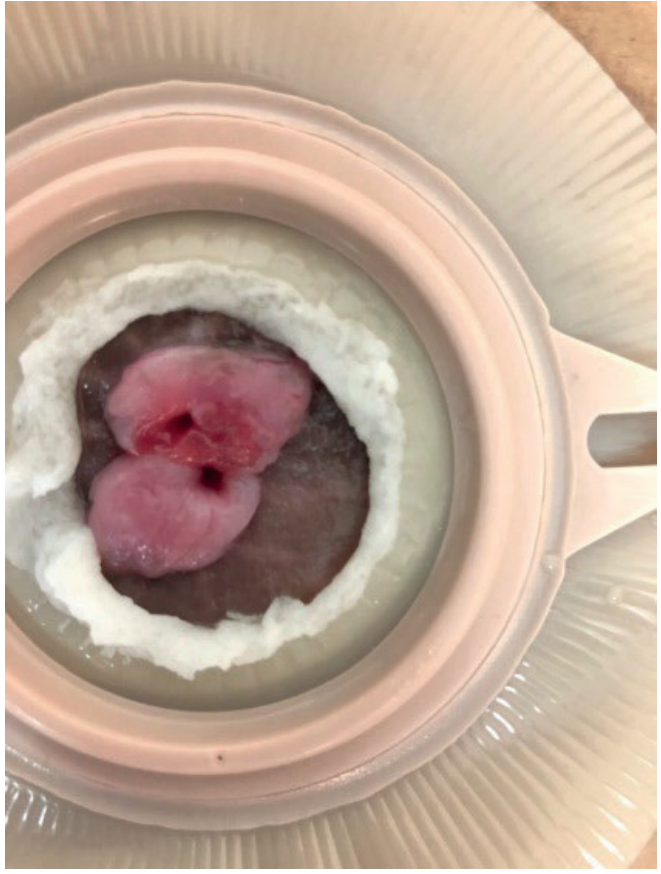

Fig. 6. Final view of the stoma after stents removal.

in the form of sandglass for ureteral separation. Under skin fat was maximally excised to shorten abdominal way tunnel and prevent fibrous changes of the ureters. To create an extraperitoneal way of the ureters 4 sides of the peritoneal window were anastomosed with rectus muscle shield. That separated the tunnel from peritoneal cavity and made it shorter, thus creating needed length. Distal ends of ureters were anastomosed with the skin in modified X-shaped technique forming single-site cutaneous ureterostomy and preventing stomal stenosis (figure 4). Ureters were everted to form the anastomosis with skin, which also allowed preventing stenosis. Two 6-7 French catheters were inserted and fixed to the skin and a stoma bag was applied (figure 5). Ureteric stents were removed 6 or 12 weeks following the surgery when the stoma was finally formed upon a surgeon's decision (figure 6).

\section{STATISTICAL ANALYSES}

Statistical analyses were performed using SPSS 22.0 (SPSS Inc. Released 2014. SPSS Statistics for Windows, Version 22.0. Chicago: SPSS Inc.). Pearson's criterion ( $\chi 2$ test) was used for categorical variables. $P$ value $<0.05$ was considered as statistically significant.

\section{RESULTS}

Main clinical characteristics of the group are summarized in Table I. Data revealed that chosen group of patients shifted towards low performance status. Major rationale for cystectomy was palliative cytoreduction. Almost half of the patients had upper tract obstruction at diagnosis with $40 \%$ of patients presenting with a decreased kidney function. 
Table I. Summary of main clinical characteristics of patients.

\begin{tabular}{cc} 
Parameter & $\begin{array}{c}\text { Modified UCS } \\
\text { (n = 89) }\end{array}$ \\
\hline Age (years), mean [IQR] & $65,5[53-75]$ \\
\hline BMI (ratio), mean [IQR] & $29,9[24,1-33,2]$ \\
\hline Preoperative upper tract obstruction, $\mathrm{n}(\%)$ & $44(49)$ \\
\hline Bladder confined cancer (pT1-T2), $\mathrm{n}(\%)$ & 9 \\
\hline Locally advanced cancer (pT3a-4a), n (\%) & 65 \\
\hline Metastatic bladder cancer (pT4b N+ M+), n (\%) & 15 \\
\hline Operative time (min), range & $194(90-360)$ \\
\hline Blood loss (ml), mean (sd) & $568(113)$ \\
\hline eGFR<60 ml/min, $\mathrm{n}(\%)$ & $36(40)$ \\
\hline ASA score (III,IV), $\mathrm{n}(\%)$ & $39(43)$ \\
\hline Follow-up (months), mean (sd) & $16(4,2)$ \\
\hline
\end{tabular}

Table II. Summary of early and late postoperative complications after UCS

\begin{tabular}{cc}
\hline & $\begin{array}{c}\text { Modified UCS } \\
\mathbf{N}=\mathbf{8 9}(\%)\end{array}$ \\
\hline Early postoperative complications & $9(10)$ \\
\hline Upper urinary tract infection & $2(2)$ \\
\hline Pneumonia & $8(9)$ \\
\hline Wound infection & $2(2)$ \\
\hline Cardiac event & $5(6)$ \\
\hline Ileus & $2(2)$ \\
\hline Thromboembolic event & - \\
\hline Anuria & $16(18)$ \\
\hline Late postoperative complications & $4(4)$ \\
\hline Stomal stricture & $2(2)$ \\
\hline Recurrent pyelonephritis & $3(3)$ \\
\hline Stone formation & $18(20)$ \\
\hline Upper tract obstruction
\end{tabular}

Surgical procedure was safe taking to account locally advanced disease in majority of patients. There were 3 intraoperative rectal injuries that were managed intraoperatively with double-suturing. Other complications included 2 major vessels and 3 ileal bowel iatrogenic trauma, which were also managed during surgery. Blood loss didn't exceeded $1000 \mathrm{ml}$ and median operation time was 194 minutes.

In early postoperative period there were 2 cardiac events and 2 thromboembolic events, with 2 deaths. Other 2 were managed conservatively and patients were discharged from the hospital with minimal delay.

Among late postoperative complications the most frequent were stomal stenosis $(\mathrm{n}=16,18 \%)$ and constipation $(n=18,20 \%)$. Stomal stenosis was managed with nephrostomy on the obstructed side (right $-n=10$, left $n=8$ ) and further re-anastomosis in 6 cases or Bricker diversion in 3. There were no cases of recurrent stomal stenosis. Constipation was mainly managed conservatively with total remission within 6 months in average. Other complications are summarized in Table II.

Average postoperative stay was 6,3 days with SD 1,2 days. In average ureteral tubes were removed after 8,2 months with SD 2,4 months. Catherter-free rate after one year of observation leveled $87 \%$. Three patients required right side stenting due to presence of hydronephrosis and no signs of stomal stenosis.

Statistical analysis of factors potentially affecting stomal stenosis didn't reveal significant dependence between ASA scoring, disease stage and preoperative upper tract obstruction. Factors that influenced postoperative obstruction were presence of CKD and increased BMI index (Table III).

\section{DISCUSSION}

Cutaneous ureterostomy remains the simplest diversion type. Historically, its construction was done retroperito-

Table III. Statistical comparison of parameters affecting stomal stenosis

\begin{tabular}{|c|c|c|c|}
\hline Parameter & Stricture presence, $n=16$ & Stricture absence, $n=73$ & Significance \\
\hline $\begin{array}{c}\text { Ureterohydronephrosis } \\
+/-\end{array}$ & $12 / 4$ & $38 / 35$ & $\begin{array}{c}p=0.09 \\
X^{2}=2.8\end{array}$ \\
\hline $\begin{array}{c}\text { ASA } \\
\text { I- II } \\
\text { III -IV } \\
\end{array}$ & $\begin{array}{l}8 \\
8 \\
\end{array}$ & $\begin{array}{l}33 \\
40 \\
\end{array}$ & $\begin{array}{l}p=0.72 \\
x^{2}=0,12\end{array}$ \\
\hline $\begin{array}{c}\text { Stage } \\
\text { II } \\
\text { III } \\
\text { IV } \\
\end{array}$ & $\begin{array}{l}4 \\
6 \\
6\end{array}$ & $\begin{array}{c}6 \\
37 \\
30 \\
\end{array}$ & $\begin{array}{c}p=0.14 \\
x^{2}=3,8\end{array}$ \\
\hline $\begin{array}{c}\text { Normal BMI } \\
\text { Obesity I - II } \\
\text { Obesity III - IV }\end{array}$ & $\begin{array}{c}1 \\
4 \\
11 \\
\end{array}$ & $\begin{array}{l}15 \\
38 \\
20 \\
\end{array}$ & $\begin{array}{l}\mathrm{p}<0.006 \\
\mathrm{X}^{2}=9.97\end{array}$ \\
\hline $\begin{array}{l}\text { CKD } \\
+/- \\
\end{array}$ & $11 / 5$ & $26 / 48$ & $\begin{array}{l}\mathrm{p}<0.013 \\
\mathrm{X}^{2}=6.14\end{array}$ \\
\hline Operation time & $\begin{array}{c}172.5 \\
{[152.5 ; 190.5]}\end{array}$ & $\begin{array}{c}180 \\
{[150 ; 220]}\end{array}$ & $\begin{array}{c}\text { Mann-Whitney U-test } \\
\mathrm{p}=0.2\end{array}$ \\
\hline Blood loss & $\begin{array}{c}450 \\
{[275 ; 600]}\end{array}$ & $\begin{array}{c}400 \\
{[300 ; 500]}\end{array}$ & $\begin{array}{c}\text { Mann-Whitney U-test } \\
p=0.41\end{array}$ \\
\hline
\end{tabular}


neally due to low surgical complexity and optimal way to prevent abdominal complications. Although retroperitoneal way remains the longest one for contralateral ureter, thus provoking high tension level, potential kinking, loss of effective distant blood supply and upper tract obstruction development. Simple bilateral ureterostomy may reduce impact of the following factors, but it also decrease life-quality, which is essential. Abdominal channel length differs from retroperitoneal by potential length, which could prevent stricture formation even in-patient with obesity. This study included only patients with unilateral stoma formation with described method, because there was only a small group of patients prior to this study, which underwent retroperitoneal or bilateral ureterocutaneostomy in our department. All statistical analysis was done within one study group, thus we tried to evaluate factors potentially effecting upper tract obstruction after surgery and complication rates.

Transperitoneal ureterostomy was mainly used in patients with advanced stage of the disease with significant co-morbidities that could affect bowel and overall complication rate. Indications to surgery were mainly derived from life-threatening and symptomatic conditions. Ureterostomy approach from one side excluded intestinal use and from the other significantly decreases operative time, that might affect length of postoperative stay. Taking to account high patients' complexity early and late postoperative complications rates were acceptable and were mainly managed conservatively. Ileal conduit (Bricker) remained an option for patients with stoma strictures or upper tract obstruction due to ureteral kinking with high success level. Postponed bowel diversion seemed to be safer after anemia and kidney function normalization. Inflammation and stone-associated conditions were not of high-grade and mainly needed conservative or minimally invasive (ureteral tube substitution or endoscopic treatment) management. Cardiac and thromboembolic events did not differ from referent literature data, thus indicating that diversion type had no systemic effect. Bowel complications stayed low due to absence of bowel stage of operation. Nevertheless, there were cases of constipation mainly derived from sigmoid colon use, which underwent conservative management with complete resolvement in average after 6 months. Overall, we received a low level of ureteral strictures thus proving methods efficacy in terms of high-risk surgical patients which would not tolerate other diversion type. Catheter free-rate 1 year after surgery showed promising results decreasing the number of planned visits and redeeming treatment-related costs.

During statistical analysis, the goal was to test clinical factors that could affect stricture formation. There were found no significant influence of age, sex, blood loss, disease stage and ASA-scoring on stoma obstruction rates. This could be explained by the fact that surgical technique and patient anatomy peculiarities might be the key influencing parameters on catheter-free rate. Further statistical evaluating revealed that presence of ureteral enlargement before surgery, high BMI and presence of CKD with high creatinine level had an effect on stricture formation.

Ureteral enlargement, from our point of view, redeems surgical complexity of skin suturing fixation and due to this fact could lead to smaller obstruction rate. Although we should also keep in mind that during obstruction ureteral microcirculation is changing, which also stays unmeasurable predictor of negative outcomes.

The other increased parameter - BMI is mainly effecting ureteral channel length due to growth of under skin fat thickness. Stoma formation becomes more complex in this patients and ureteral length is crucial. Transperitoneal diversion helps to downsize length of the intra-abdominal channel and save it for extra abdominal part. In our opinion, excision of fat during stoma formation remains controversial in terms of provoking fibrous stoma transformation, so its use is probably efficient only for increasing surgical convenience. Described diversion type remains safe even in patients with obesity.

Concerning CKD probably it only proves the theory of safe surgery in co-morbid patients, although obstruction occurred in time, there were no severe bowel related complications that could have been fatal and increase post-surgical mortality rate. Decreased kidney filtration leads to worse tissue regeneration, thus necrotic and fibrously transformed distal of the ureter seems quite logic. Despite stenosis possibility in CKD patients, modified cutaneostomy preserves patient's safety and could be a method of choice.

The last important fact dedicated to this surgery type, is that, because ureter passes its abdominal way beneath the visceral peritoneum of the sigmoid colon, it remains out of the abdominal cavity. This means that even in cases when urine leakage occurs, it remains separated and will not lead to peritonitis. Although in this cases, probability of obstruction may raise.

Vast progress in therapeutic and urgent care leads to an increased possibility for conducting surgery in co-morbid and surgically complex patients. Choice of diversion type is obvious in young patients with no severe concomitant pathology, although things can radically change in hereditary, symptomatic and advanced stage cases. Implementation of ureterostomy into daily urological practice is mainly associated with a search for treatment options in clinical decision-making. Transperitoneal approach seems quite feasible and tends to improve surgical outcomes.

\section{CONCLUSIONS}

Unilateral transperitoneal cutaneostomy using mesosigmoid colon is the surgery of choice in advanced bladder cancer patients. Major advantages are simplicity of the technique, minimization of bowel complication risks and short recovery after surgery. Described method could be a useful option for practicing urologist regarding its safety and benefits. Further multi-center investigation is needed to prove its efficacy.

\section{REFERENCES}

1. Lee R., Abol-Enein H., Artibani W., et al. Urinary diversion after radical cystectomy for bladder cancer: options, patient selection and outcomes. BJU. 2014; 113: 11-23 doi:10.1111/ bju.12121 
2. GaméX., Soulié M., Seguin P., et al. Radical Cystectomy in Patients Older than 75 Years: Assessment of Morbidity and Mortality. European urology. 2001; 39(5): 525-529.

3. Kozacıoğlu Z., Değirmenci T., Günlüsoy B., et al. Ureterocutaneostomy: for whom and when? Turkish Journal of Urology. 39(3):143-6. doi:10.5152/tud.2013.030

4. Yadav P., Mittal V., Gaur P., et al. A modified cutaneous ureterostomy provides satisfactory short and midterm outcomes in select cases. Turk J Urol. 2018; 44(5): 399-405. Doi:10.5152/ tud.2018.Turk J Urol 51437

5. Hatakeyama S., Koie T., Narita T., et al Renal Function Outcomes and Risk Factors for Risk Factors for Stage 3B Chronic Kidney Disease after Urinary Diversion in patients with muscle invasive bladder cancer. PLOS One. 2016; 11(2). D0l:10.1371/journal.pone.0149544

6. Chitale S., Chitale V. Bilateral ureterocutaneostomy with modified stoma: long-term follow-up World J Urol. 2006; 24: 220-223. doi.10.1007/s00345-006-0080-4

7. Terai A., Yoshimura K., Ueda N., et al. Clinical outcome of tubeless cutaneous ureterostomy by the Toyoda method AKITO. International Journal of Urology. 2006; 13: 891-895.

8. Furubayashi N., Negishi T., Kashiwagi E., et al. Clinical outcome of transperitoneal ureterocutaneostomy using the transverse mesocolon Molecular and Clinical Oncology. 2013; 1: 721-725.

9. Tsaturyan A., Sahakyan S., Muradyan A., et al. A new modification of tubeless cutaneous ureterostomy following radical cystectomy. Int Urol Nephrol. 2019; 51: 959-967. doi: 10.1007 Int Urol Nephrol / 11255019-02145
This work was done as a part of Scientific Research "Improvement of indications and strategy of combination treatment of patients with metastatic and advanced bladder cancer", funded by Ministry of Health of Ukraine.

\section{ORCID and contributionship:}

Stakhovskyi E. Oleksandr - 0000-0002-0030-5459 A,D,E

Pikul V. Maksym - 0000-0003-1590-235X ${ }^{A, D}$

Semko L. Sofiya - 0000-0002-1657-5752 ${ }^{B, C}$

Voylenko A. Oleg - 0000-0003-3781-3622 ${ }^{\mathrm{C}}$

Kononenko A. Oleksii - 0000-0002-7081-5964 ${ }^{\text {B,C }}$

Vitruk V. Iurii - 0000-0003-3466-0552 ${ }^{\text {B,C }}$

Grechko O. Bogdan - 0000-0002-2847-3980 ${ }^{\text {B,E }}$

Stakhovsky O. Eduard - 0000-0001-5677-8768 E,F

\section{Conflict of interest:}

The Authors declare no conflict of interest

\section{CORRESPONDING AUTHOR} Stakhovskyi E. Oleksandr

Department of Plastic and Reconstructive Oncological Urology, National Cancer Institute, 33/43 Lomonosova Str., 03022, Kyiv, Ukraine tel: +380503817217

e-mail: stakhovsky9@gmail.com

Received: 27.01 .2020

Accepted: 30.03 .2020

A - Work concept and design, B - Data collection and analysis, C - Responsibility for statistical analysis, D - Writing the article, $\mathbf{E}$-Critical review, $\mathbf{F}$ - Final approval of the article 